\section{Fiber ringdown temperature sensors}

\author{
Chuji Wang \\ Mississippi State University \\ Diagnostic Instrumentation and Analysis \\ Laboratory \\ 205 Research Boulevard \\ Starkville, Mississippi 39759 \\ E-mail: wang@dial.msstate.edu
}

\begin{abstract}
A new method of developing fiber temperature sensors using a fiber Bragg grating-loop ringdown scheme is introduced. With this new technique, temperature measurements are converted to measuring time constants. Temperature sensing up to $593^{\circ} \mathrm{C}$ has been demonstrated using a proof-of-concept device. The sensor's stability, repeatability, sensitivity, and dynamic range are also explored. (c) 2005 Society of Photo-Optical Instrumentation Engineers. [DOI: 10.1117/1.1869512]
\end{abstract}

Subject terms: fiber Bragg grating; fiber loop ringdown; temperature sensors.

Paper L040601 received Sep. 2, 2004; revised manuscript received Dec. 17, 2004; accepted for publication Dec. 23, 2004; appeared online Jan. 3, 2005; published online Mar. 4, 2005.

\section{Introduction}

Combining optical fiber components with advanced laser spectroscopic techniques may bring huge application potential in sensor development. Very recently, we reported a new type of fiber pressure sensors, which adopt a cavity ringdown concept ${ }^{1}$ and convert pressure measurements into a time-domain scheme, measuring ringdown times to determine pressures. ${ }^{2,3}$

In this letter, we describe a new type of fiber temperature sensors using a fiber Bragg grating-loop ringdown (FBG-LRD) technique and demonstrate its potential for temperature sensor development.

Fiber loop ringdown was initially introduced for trace chemical species detections. ${ }^{4-6}$ A laser beam is coupled into the fiber loop, and when the light source is rapidly shut off, the resultant light rings inside the fiber loop for many round trips. In each round trip, a small fraction of light leaks into a photodetector through a fiber coupler. The rest of the light rings in the fiber experience internal fiber transmission losses. The signal intensity observed by the detector follows an exponential decay: the lower the optical losses, the longer the decay time constant (ringdown time). When a FBG is incorporated into a fiber loop, additional fiber transmission losses, attributed to the fiber spliced insertion loss and the FBG induced insertion loss, are added to the fiber loop. Therefore, the temperature sensitive optical transmission loss occurring in the FBG can be measured through monitoring the ringdown time.

\section{Experiment and Results}

The fiber Bragg grating-loop ringdown device mainly consists of a FBG which is written in a section of single mode

0091-3286/2005/\$22.00 @ 2005 SPIE fused silica fiber, two identical $2 \times 1$ fiber couplers, two sections of fused silica single mode fiber (Corning SMF 28), a temperature controlled distributed feedback diode laser (NEL America, NLK1U5E1AA, $1567 \mathrm{~nm}$ ), and an InGaAs photodetector (PD 400, Thorlabs). The quoted split ratio in the two-leg end of the fiber couplers is 1:99. The two one-leg ends of the couplers are spliced together there two $99 \%$ legs of the two couplers are separately spliced with the two ends of the fiber on which the grating is written, forming a fiber Bragg grating loop. The light from the single mode fiber of the pig-tailed laser diode is coupled into the fiber loop through the $1 \%$ leg with a FC/APC fiber connector. The $1 \%$ leg of the second coupler is coupled to the photodetector. The total length of the loop is $65 \mathrm{~m}$. The quoted reflectance of the grating at the central wavelength $(1567 \mathrm{~nm})$ is $83.9 \%$ (corresponding to the light transmission loss of $0.76 \mathrm{~dB}$ ) with a bandwidth of $0.25 \mathrm{~nm}$. The quoted insertion loss of each coupler is less than $0.2 \mathrm{~dB}$. The typical fiber-to-fiber spliced insertion loss is $0.03 \mathrm{~dB}$.

Figure 1 shows a typical fiber Bragg grating-loop ringdown waveform. The contour of the decay waveform was fitted to a single exponential decay with a ringdown time of $4.4 \mu \mathrm{s}$. At a given temperature and a fixed laser wavelength, the ringdown time $\tau$ is a constant, which characterizes the physical properties of the sensor, such as the total length of the loop, the refractive index, the total insertion loss, and the grating-induced insertion loss. ${ }^{2,3}$ The time interval between two adjacent spikes shown in the waveform is the round trip time of the laser inside the loop. If both the refractive index and the length of the fiber loop are known, the recorded ringdown time can be used to characterize the wavelength-dependent bandwidth curve of the FBG.

Figure 2 shows a typical response of the ringdown time to the change in grating temperature. The temperature change was controlled by heating and nonheating the section of fiber which contains the FBG. In the experiment, this section of fiber was taped on the surface of a compact disk so that there was no bending or stress when the grating was heated by a heat gun. Figure 2 shows that the ringdown time dropped from 4.4 to $3.1 \mu$ s when the grating was heated and the ringdown time increased back to $4.4 \mu \mathrm{s}$ when the heating was removed. Each data point in Fig. 2 was generated from averaging 50 ringdown events and the frequency of the ringdown event was $10 \mathrm{~Hz}$. In the processing of each individual heating and nonheating, the distance

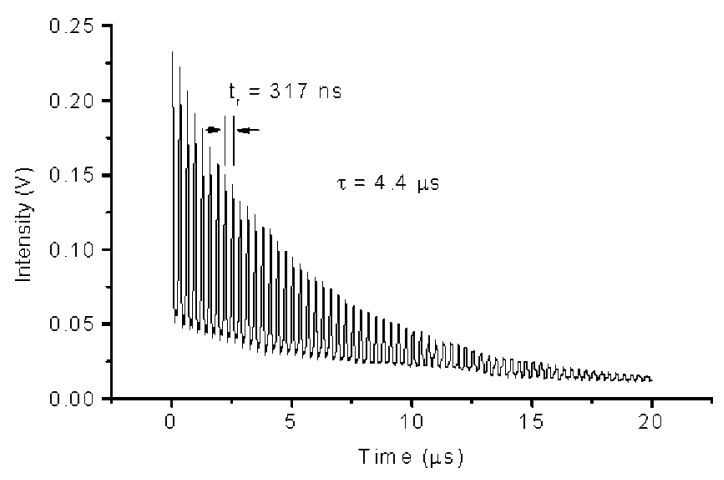

Fig. 1 Typical fiber Bragg grating-loop ringdown waveform. The ringdown waveform was recorded with a laser wavelength of 1568.2 $\mathrm{nm}$ and a FBG temperature of $24^{\circ} \mathrm{C}$. 


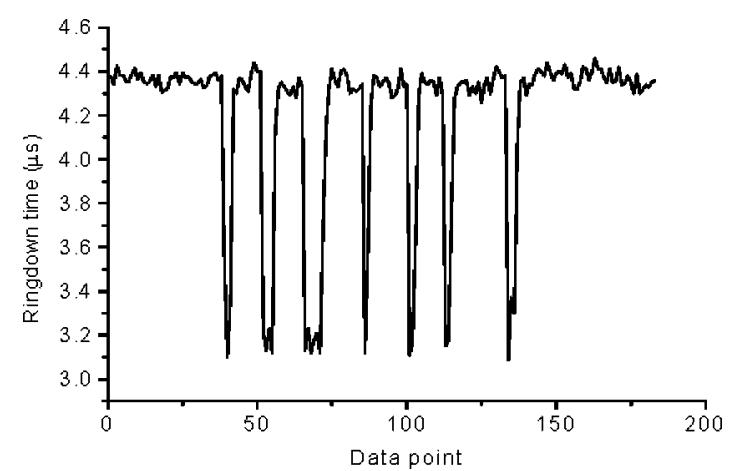

Fig. 2 Fiber Bragg grating-loop ringdown temperature sensor demonstrates a rapid response and good repeatability.

of the heat gun head to the grating was kept at approximately $5 \mathrm{~cm}$. Each individual heating time was not intentionally controlled to be the same, but within the range of 5 to $20 \mathrm{~s}$. The data in Fig. 2 clearly show that the temperature sensor has fast response and good repeatability.

To examine the sensor's performance, the laser wavelength was first tuned to $1568.2 \mathrm{~nm}, 1.2 \mathrm{~nm}$ away from the grating's central wavelength. The recorded ringdown time was $4.4 \mu \mathrm{s}$ at room temperature, $24^{\circ} \mathrm{C}$. With the increase of the FBG temperature, the whole bandwidth curve shifts, and the resultant optical transmittance of the grating at $1568.2 \mathrm{~nm}$ changes. Equivalently, the light transmission loss in the FBG changes. Therefore, the observed ringdown times differ. It should be noted that compared with the change of the FBG-induced transmission loss, the change of the fiber absorption loss and scattering loss resulting from the temperature-induced thermal expansion occurring in the section of the fiber $(20 \mathrm{~mm})$ on which the FBG is written is negligible. In the experiment, the section of fiber containing the FBG was immersed into a water bath heated by an electrical heater. The FBG temperature was monitored by a glass thermometer (NESLAB, 1508/1509, $24-$ $76^{\circ} \mathrm{C}$ ) with a graduation of $0.1^{\circ} \mathrm{C}$. The probe of the thermometer was deployed close to the grating in the bath, so that the reading temperature in the thermometer indicated the actual temperature of the FBG. Figure 3 shows that the ringdown time dropped from 4.4 to $3.5 \mu$ s when the FBG

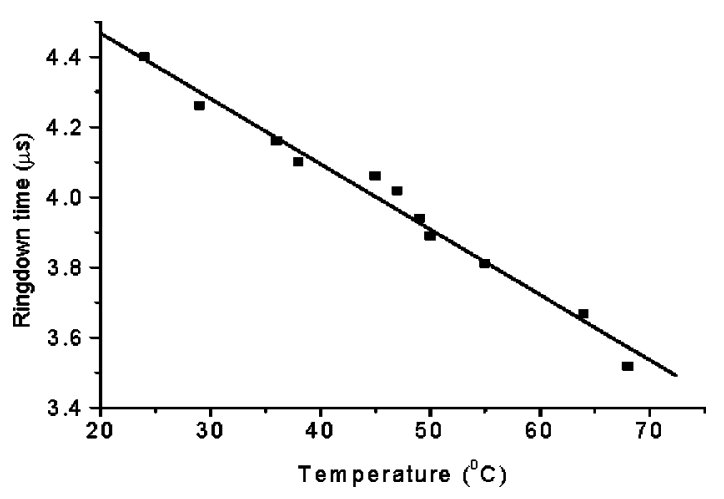

Fig. 3 Ringdown time responses to the change of the FBG temperature. The dots and the line represent the experimental data and the fitted curve, respectively. temperature changed from $24^{\circ} \mathrm{C}$ to $68^{\circ} \mathrm{C}$ and that the sensor's response had a good linearity in the temperature range examined. To explore a larger measuring dynamic range of the sensor, another laser diode was used, which provided a laser output at $1572 \mathrm{~nm}$, far away from the central wavelength of the FBG, $1567 \mathrm{~nm}$. The light transmittance rate of the FBG at this wavelength is insensitive to the wavelength shifting resulting from a change of the FBG temperature. Although the detection sensitivity is relatively low at this wavelength, the upper limit of the sensor's measuring range drastically increases. When the FBG was heated by the heat gun from $24^{\circ} \mathrm{C}$ to $593^{\circ} \mathrm{C}$ (the maximum thermal equilibrium temperature of the heat gun used) the observed change in ringdown time was only $0.8 \mu$ s. This suggests that the measuring dynamic range of such a sensor can be specifically designed by selecting different laser sources and/or FBGs with different specifications such as the bandwidth, transmittance, and feature of the bandwidth curve.

Similarly, when the light wavelength was tuned to $1567.9 \mathrm{~nm}$, the sensor became the most sensitive since a small shift of the bandwidth curve results in a large change of the transmittance rate at this wavelength. The increase of the temperature by $10^{\circ} \mathrm{C}$ in the range of $24-50^{\circ} \mathrm{C}$ resulted in a $1-\mu \mathrm{s}$ decrease in the ringdown time. The standard deviation $(\sigma)$ of the ringdown time averaging $(\bar{\tau})$ from 50 ringdown events was $0.02 \mu \mathrm{s}$. This implies that the detection sensitivity of the temperature measurement using such a device is around $0.2^{\circ} \mathrm{C}$ based on one $\sigma$ criteria. The sensor's stability depends on the ringdown baseline stability, which is defined as $\sigma / \bar{\tau}$. Averaging over 50 ringdown events yielded the baseline stability of $0.45 \%$, which is approximately equivalent to the sensor's stability of the temperature sensing, e.g., $4.5^{\circ} \mathrm{C}$ drifting in a $1000^{\circ} \mathrm{C}$ base.

\section{Conclusions}

The fiber Bragg grating-loop ringdown technique is introduced to develop fiber temperature sensors. Temperature sensing up to $593^{\circ} \mathrm{C}$ has been demonstrated using the proof-of-concept device. Potential advantages of this new type of temperature sensors compared with the current generation FBGs temperature sensors are the higher sensitivity, larger measuring dynamic range, and lower costs.

\section{Acknowledgments}

The author is thankful for the support through the Cooperative Agreement between the United States Department of Energy and the Diagnostic Instrumentation and Analysis Laboratory at Mississippi State University.

\section{References}

1. A. O'Keefe and D. A. G. Deacon, "Cavity ring-down optical spectrometer for absorption measurements using pulsed laser sources," Rev. Sci. Instrum. 59, 2544-2551 (1988).

2. C. Wang and S. T. Scherrer, "Fiber ringdown pressure sensors," Opt. Lett. 29, 352-354 (2004).

3. C. Wang and S. T. Scherrer, "Fiber loop ringdown for physical sensor development: pressure sensor," Appl. Opt. 43(35), 6458-6464 (2004).

4. G. Stewart, K. Atherton, H. Yu, and B. Culshaw, "Investigation of an optical fibre amplifier loop for intracavity and cavity ring-down loss measurements," Meas. Sci. Technol. 12, 843-849 (2001).

5. R. S. Brown, I. Kozin, Z. Tong, R. D. Oleschuk, and H.-P. Loock, "Fiber-loop ring-down spectroscopy," J. Chem. Phys. 117, 1044410447 (2002).

6. P. B. Tarsa, P. Rabinowitz, and K. K. Lehmann, "Passive optical fiber resonator for cavity ring-down spectroscopy," Abstracts of Papers, 224th ACS National Meeting, Boston, MA (2002). 